\title{
An Accurate and Efficient Bayesian Method for Automatic Segmentation of Brain MRI
}

\author{
J.L. Marroquin ${ }^{1}$, B.C. Vemuri ${ }^{2}$, S. Botello ${ }^{1}$, and F. Calderon ${ }^{3}$ \\ 1 Centro de Investigación en Matemáticas \\ Apdo. Postal 402 Guanajuato, Gto. 36000, Mexico \\ 2 Dept. of Computer \& Information Science \& Engineering \\ University of Florida \\ Gainesville, Fl. USA. 32611-6120 \\ 3 Division of Estudios Postgrado \\ Universidad Michoacana de San Nicolas de Hidalgo \\ Santiago Tapia 403. Colonia Centro \\ Morelia, Michoacan, Mexico
}

\begin{abstract}
Automatic 3D segmentation of the brain from MR scans is a challenging problem that has received enormous amount of attention lately. Of the techniques reported in literature, very few are fully automatic. In this paper, we present an efficient and accurate, fully automatic 3D segmentation procedure for brain MR scans. It has several salient features namely, (1) instead of a single multiplicative bias field that affects all tissue intensities, separate parametric smooth models are used for the intensity of each class. This may be a more realistic model and avoids the need for a logarithmic transformation. (2) A brain atlas is used in conjunction with a robust registration procedure to find a nonrigid transformation that maps the standard brain to the specimen to be segmented. This transformation is then used to: segment the brain from non-brain tissue; compute prior probabilities for each class at each voxel location and find an appropriate automatic initialization. (3) Finally, a novel algorithm is presented which is a variant of the EM procedure, that incorporates a fast and accurate way to find optimal segmentations, given the intensity models along with the spatial coherence assumption. Experimental results with both synthetic and real data are included, as well as comparisons of the performance of our algorithm with that of other published methods.
\end{abstract}

\section{Introduction}

Image segmentation is a fundamental problem in image processing and computer vision with numerous applications including but not limited to medical image analysis, image compression, etc. Three-dimensional processing and visualization of medical images is a rapidly growing area of research and MRI has provided a means for imaging tissue at very high resolutions providing the desired information for use in fields like radiotherapy treatment planning, stereotactic neurosurgery and others. In the context of neuro-imaging, 3-D segmentation of 
white matter, gray matter, CSF, bone etc. is extremely important for quantitative analysis such as volume measurements. It has already been established that volumetric analysis of different parts of the brain is useful in assessing progress or remission of various diseases e.g., the Alzheimer's and Epilepsy.

The main difficulties found in the automatic segmentation of MR brain images derive from the fact that image intensities are not necessarily constant for each tissue class. Thus, traditional clustering schemes, such as those reported in 6 17, or methods based on thresholding [32 do not yield desired results. An additional difficulty stems from the presence of noise in the data, which causes pixel-wise classification methods to give unrealistic results, where the regions corresponding to each tissue class may appear granular, fragmented, or violating anatomical constraints. Thus, it is necessary to devise methods that simultaneously include the estimation of variable intensity models for each class, and prior knowledge about the location and spatial coherence of the corresponding regions.

The most natural framework for the design of such methods is probabilistic, and involves the simultaneous estimation of a discrete field (the tissue class) and a continuous one (the corresponding intensities). Spatial coherence assumptions may be incorporated using a Bayesian approach, in the form of prior Markov Random Field (MRF) models [28,21], and anatomical constraints about the location of each class may be specified as prior probabilities, obtained from statistical studies [23]. The resulting estimation procedure is iterative, and consists, in general, of 2 steps that are repeated until convergence: (i) Estimate the most likely segmentation, given the intensity models for each class. (ii) Estimate the intensity models, given a segmentation, with an appropriate initialization step.

If a "soft" segmentation is computed in the first step, i.e., if one computes the marginal probabilities for each tissue class, this iterative procedure is equivalent to the Expectation maximization (EM) algorithm [2], and it has been used by a number of researchers $10730 \mid 111922,23$. The efficiency of these methods, however, has been limited by several factors. If no spatial coherence assumptions are included, the estimation of the posterior marginal probabilities for each class at each voxel is straightforward and computationally efficient (as in 11]); the quality of this type of segmentation, however, degrades rapidly if noise is present in the data. If, on the other hand, spatial coherence assumptions are included, the exact computation of the optimal segmentation becomes intractable, so that approximations must be made. The most precise are based on Markov Chain Monte Carlo (MCMC) methods, such as the Gibbs Sampler or Metropolis algorithms 28, but are computationally very expensive. Deterministic approximations, such as the ones based on Mean Field (MF) Theory [15]9,20,31] (as used in [23]) are faster, although less precise and more vulnerable to noise.

As an alternative, one can estimate a hard segmentation in the first step. Here, one has to choose an appropriate cost function, to get the corresponding optimal estimator (e.g., the Maximum a Posteriori (MAP) or the Maximizer of the Posterior Marginals (MPM) estimators). It was shown in [13], that for low SNR (signal to noise ratio), the MPM criterion yields superior segmentation re- 
sults whereas for high SNR, both are more or less equivalent. Another important issue to be taken into consideration is: for a given criterion, what is the best algorithm for finding the optimal estimator? Computational efficiency is a crucial issue that influences the answer to this question. Again, in the presence of immense computational resources, MCMC schemes (such as Simulated Annealing 29] as used in [18 for computing the MAP) are very apt. On the other hand, if computational efficiency is of high priority, it is necessary to resort to viable approximations, such as the ICM algorithm 3] used in 26,18,16] for computing the MAP, which is very fast, but highly vulnerable to noise. It is also possible to use the MF or the Gauss-Markov Measure Field (GMMF) 14] approximations for computing the MPM. In [14], it is shown that for low SNR, GMMF is found to be superior to MF, both in terms of accuracy and of computational efficiency. For medium-high SNR MF and GMMF give equivalent results, but GMMF is faster.

The computation of the image intensities associated with each tissue class for a given segmentation (step 2) is complicated by the fact that both intrascan and interscan intensity inhomogeneities often appear, due to poor radio frequency coil uniformity, operating conditions of the MR equipment, etc. These inhomogeneities are usually modeled as a single multiplicative degradation (bias field) that affects a constant intensity model for each class 111916,2223 . Thus, the intensity associated to class $k$ at voxel $r$ is usually modeled as: $I_{k}(r)=\beta(r) \mu_{k}$, where the bias field $\beta$ is assumed to have a slow spatial variation, and $\mu_{k}$ denotes the unknown constant intensity for class $k$. This intensity model, however, may not be very realistic: there may be variations in the magnetic susceptibility of the tissues; attenuation may be different depending on the location of anatomical structures, etc. To compensate for these effects, in [18 a Parzen-window distribution is used to model the conditional probability of the intensity for each tissue class. This distribution, however, requires of high level user intervention to be defined in each case, and leads to equations that are, in practice, too complicated to be solved exactly.

An additional complication introduced by the model described above is that to make it computationally tractable, the multiplicative degradation must be transformed into an additive one by means of a logarithmic transformation, which has the undesirable effect of altering the intensity distribution, making the tissue separation more difficult. A further difficulty comes from the fact that non-brain tissue may be wrongly classified as gray or white matter in the segmentation step, leading to wrong estimated values for the class intensities. This may be avoided if the brain/non-brain separation is effected prior to segmentation, either manually (as in 16]), or automatically, using for example, active contours [24, active brain templates [16] or registering a standard brain atlas [23].

In summary, while the Bayesian approach to MRI segmentation appears to be very promising, there are several problems which limit its performance and need resolution prior to making it a feasible approach. Below is an itemization of these problems (not in any specific order). (1) If spatial coherence assumptions 
are included, an exact computation of the optimal segmentation at each step becomes intractable and approximations are either computationally expensive or inaccurate. (2) A single bias field affecting all tissue classes equally may not be realistic. (3) A multiplicative bias model requires a logarithmic transformation that distorts the intensity distribution. (4) Computation of the intensity models for the tissue classes is made rather difficult by the presence of misclassified nonbrain tissue. (5) Appropriate initialization of the iterative procedure is difficult to find in a fully automatic way.

The goal of this paper is to present a fully automatic Bayesian-based scheme that overcomes these difficulties, and thus, has superior performance. To achieve this, our method incorporates the following features: (i) Instead of a single multiplicative bias field that affects all tissue intensities, we propose separate parametric, smooth models for the intensity of each class. This may be a more realistic model and avoids the need for a logarithmic transformation and hence the related nonlinear distortions. (ii) We propose the use of a brain atlas (as in [23]), together with a robust registration procedure to find a non-rigid transformation that maps the standard brain to the specimen to be segmented. This transformation is then used to segment the brain from non-brain tissue; compute prior probabilities for each class at each voxel location and find an appropriate automatic initialization. (iii) We propose a novel variant of the EM algorithm [2] which allows for the use of a fast and accurate way to find optimal segmentations, given the intensity models which incorporate the spatial coherence assumptions.

The rest of this paper is organized as follows: the above described algorithm features are elaborated upon in section 2. Section 3 contains the experimental validation of the complete procedure, and finally some conclusions are drawn in section 4 .

\section{An Improved Bayesian Approach to MRI Segmentation}

In this section, we first present a Bayesian formulation of the segmentation problem followed by a novel, accurate and efficient 3D segmentation algorithm which is dubbed as the MPM-MAP algorithm.

\subsection{Bayesian Formulation of the Segmentation Problem}

In a Bayesian approach to segmentation, one specifies the distribution of the observation noise, and introduces the prior constraint about the spatial coherence of the support regions in the form of a prior probability distribution on the set of possible segmentations. To describe this approach, we introduce the following notation: let $L$ denote the pixel lattice, and $\{g(r), r \in L\}$ denote the observed images; let $\left\{\Phi\left(r ; \theta_{k}\right), k=1, \ldots, K\right\}$ denote a set of models characterized by the parameter vector $\theta=\left(\theta_{1}, \ldots, \theta_{K}\right)$, which describe the variation of the value of a property $f$ over $L$. Note that $f$ may represent a scalar valued data representing the intensity values in a $3 \mathrm{D}$ volume, or it may be a vector valued function defined on a $3 \mathrm{D}$ image grid. Each model $k$ is supposed to be valid in a region $R_{k} \subseteq L$ 
- note that $R_{k}$ is a partitioning of $L$ - so that the value of the field $f$ at pixel $r$ is $f(r)=\sum_{k=1}^{K} \Phi\left(r ; \theta_{k}\right) b_{k}(r)$, where $b_{k}(r)$ is the indicator function for region $R_{k}$, i.e., $b_{k}(r)=1$ if $r \in R_{k}$, and $b_{k}(r)=0$, otherwise. Assuming a Gaussian observation noise model, the likelihood of the data given the models and the support regions is :

$$
P(g \mid b, \theta)=\frac{1}{Z_{L}} \Pi_{r \in L} \Pi_{k=1}^{K} l_{k}(r)^{b_{k}(r)}
$$

where $Z_{L}$ is a constant and

$$
\begin{aligned}
l_{k}(r) & =\mathbf{\Phi}\left(g(r) \mid \theta, b_{k}(r)=1\right) \\
& =\sqrt{\frac{\gamma}{\pi}} \exp \left[-\gamma\left|g(r)-\Phi\left(r, \theta_{k}\right)\right|^{2}\right]
\end{aligned}
$$

where $\gamma$ is a parameter that depends on the noise variance. One may also have prior probabilities that specify the probability of the voxel at location $r$ belonging to each of the classes. We will denote these probabilities by $q_{k}(r)=\mathbf{\Phi}$ (voxel $r \in$ class $k$ ). The constraint for the spatial coherence of the support regions $\left\{R_{1}, \ldots, R_{K}\right\}$ may be expressed, in probabilistic terms, in the form of a prior Markov Random Field (MRF) model on the indicator variables $b$; in particular, one may assume a prior generalized Ising (Potts) model [13, where the neighborhood $N_{r}$ of each site $r \in L$ is formed only by its nearest neighbors (in a 2-D image there are 4 such neighbors, and in a 3-D volume image there are 6 in the simplest neighborhood system). This gives the prior Gibbs distribution: $P_{b}(b)=\frac{1}{Z_{b}} \exp \left[\beta \sum_{<r, s>} V(b(r), b(s))\right]$. Where, $Z_{b}$ is a normalizing constant, $\beta$ is a positive parameter controlling the granularity of the regions, the summation is performed over all nearest-neighbor pairs of sites in $L$ and the Ising potential $V_{I}$ is given by,

$$
V_{I}(b(r), b(s))= \begin{cases}-1 & , \text { if } \sum_{k=1}^{K} b_{k}(r) b_{k}(s)=1 \\ 1, & \text { otherwise }\end{cases}
$$

(note that $b_{k}(r) \in\{0,1\}$ and that $\sum_{k=1}^{K} b_{k}(r)=1$ for all $r \in L$ ). The posterior distribution is computed using Bayes rule and is of the form: $P(b, \theta \mid g)=$ $\frac{1}{Z} \exp [-U(b, \theta)]$, where $Z$ is a normalizing constant and

$$
\begin{aligned}
U(b, \theta)= & -\sum_{r \in L} \sum_{k=1}^{K}\left[b_{k}(r) \log l_{k}(r)+\log q_{k}(r)\right] \\
& +\beta \sum_{<r, s>} V_{I}(b(r), b(s))+\log P(\theta)
\end{aligned}
$$

where $l_{k}(r)$ is given by Eq. (11), and $P(\theta)$ represents the prior distribution of the parameters that define each intensity model. In the classical Expectation Maximization (EM) approach [2], the $b$ variables are considered "missing data" and $U$ is minimized in a 2-step procedure consisting of an (E) step in which one 
first computes the expected value $\langle b\rangle$ of the $b$ variables, given an estimate of the model parameters $\theta$, while in the second (M) step, one minimizes the $U$ function, where the $b$ variables are replaced by their expected value. The drawback of this approach lies in the computational complexity associated with the computation of $\langle b\rangle$ : since the variables are binary, their expected value is equal to the posterior marginal probabilities given by

$$
\pi_{k}(r)=\mathbf{\Phi}\left(b_{k}(r)=1 \mid \theta, g\right)=\sum_{b: b_{k}(r)=1} \frac{1}{Z} \exp [-U(b, \theta)]
$$

It is clear that the summation in (4) has too many terms to be exactly computed, so it has to be approximated. There are several methods that have been proposed for estimating the posterior marginals of discrete--valued MRF's: stochastic approaches based on the construction of regular Markov chains [28,13], and deterministic approaches based on the mean-field approximation [915] in which the estimated marginals are obtained as solutions of large systems of coupled non-linear equations. These approaches, however, are computationally very expensive. Other methods [20,31] that also involve mean field approximations using different cost functions require solution to a large coupled system of nonlinear equations but possibly lead to more accurate - than the approximation outlined in [15] - approximation of the marginals. In this paper, we propose a novel approach with which one can get fast, high quality estimators solving a set of decoupled, linear systems of equations. It is based on the fact that, given a Gibbs distribution with energy of the form:

$$
U(b)=\sum_{r \in L} V_{0}(b(r))+\beta \sum_{<r, s>} V_{I}(b(r), b(s))
$$

where the $b$ 's are random $K$-vectors satisfying: $b_{k}(r) \in\{0,1\}$ and $\sum_{k=1}^{K} b_{k}(r)=$ 1 for all $r \in L$, and $V_{I}$ is an Ising potential, then, the marginals $\pi_{k}(r)=\mathbf{\Phi}\left(b_{k}(r)=\right.$ 1) may be approximated by the expected value of random vectors $p$ that admit a Gibbsian model with energy of the form:

$$
U_{p}(p)=\sum_{r \in L}|p(r)-\widehat{p}(r)|^{2}+\lambda \sum_{<r, s>}|p(r)-p(s)|^{2}
$$

where $\widehat{p}_{k}(r)=\frac{1}{Z} \exp \left[-V_{0}\left(e_{k}\right)\right]$ with $Z$ a normalizing constant and where $e_{k}$ is a unit vector with a 1 in the $k^{\text {th }}$ position. (see [14] for details). Therefore, an approximation to the marginals may be constructed by minimizing (5) , that is, by solving $K$ sets of decoupled linear equations, that result from setting the partial derivatives of $U_{p}$, with respect to the variables $p_{k}(r)$, equal to zero.The EM algorithm, however, may be quite sensitive to errors in the estimated marginals. Therefore, if this fast approximation procedure is to be used, it is necessary to modify the algorithm to make it more robust with respect to this kind of errors. This modified algorithm will be described in the next subsection. 


\subsection{The MPM-MAP Algorithm}

The algorithm we propose for the simultaneous estimation of $b$ and $\theta$ is based on Bayesian estimation theory. In this approach, we consider $b$ and $\theta$ as random vectors whose optimal estimators are to be found via the minimization of the expected value of an appropriate cost function, taken with respect to the posterior distribution. The cost function we propose is:

$$
C(\widehat{b}, \widehat{\theta}, b, \theta)=1-\delta(\theta-\widehat{\theta})+\frac{1}{|L|} \sum_{r \in L}[1-\delta(b(r)-\widehat{b}(r))]
$$

where the $\delta$ functions equal 1 if their argument is the 0 vector, and equal 0 otherwise. The first term requires that the estimated parameter vector $\widehat{\theta}$ be on the average - a perfect estimator, while the second term requires that the estimated indicator functions $\widehat{b}$ for the support regions, minimize the expected number of segmentation errors. Denoting $Q(\widehat{b}, \widehat{\theta})=E[C(\widehat{b}, \widehat{\theta}, b, \theta)]$, the optimal estimators $\left(\widehat{b}^{*}, \widehat{\theta}^{*}\right)$ are, therefore,

$$
\left(\widehat{b}^{*}, \widehat{\theta}^{*}\right)=\arg \min _{\widehat{b}, \widehat{\theta}} Q(\widehat{b}, \widehat{\theta})
$$

To minimize $Q$ we propose a 2 -step procedure in which $Q(\widehat{b}, \widehat{\theta})$ is minimized with respect to $\widehat{b}$ for a given $\widehat{\theta}$ in a first step, and then minimized with respect to $\widehat{\theta}$, keeping the optimal $\widehat{b}$ fixed, in the second step. To derive the implementation of the first step, we make the following considerations: suppose that $\widehat{\theta}=\bar{\theta}$ is given. The optimal estimator for $\widehat{b}$ is found by minimizing the expected value of the second term of Eq. (6) :

$$
\begin{aligned}
& \sum_{b} \sum_{r \in L}[1-\delta(b(r)-\widehat{b}(r))] P(b, \bar{\theta} \mid g) \\
= & |L|-\sum_{r \in L} \sum_{k=1}^{K} \pi_{k}(r) \widehat{b}_{k}(r)
\end{aligned}
$$

where

$$
\pi_{k}(r)=\sum_{b: b_{k}(r)=1} P(b, \bar{\theta} \mid g)
$$

is the posterior marginal probability for the support region $k$ at pixel $r$. Expression (7) is minimized by setting $\widehat{b}=\bar{b}$, where $\bar{b}_{k}(r)=1$, if $\pi_{k}(r)>\pi_{k^{\prime}}(r)$ for $k^{\prime} \neq k$, and $\bar{b}_{k}(r)=0$, otherwise. This estimator is called the Maximizer of the Posterior Marginals or MPM estimator [13 for $b$ given $\bar{\theta}$.

To minimize $Q$ with respect to $\widehat{\theta}$ for a fixed $\widehat{b}=\bar{b}$ one needs to consider only the expected value of the first term of ([6), so that the optimal (Maximum a Posteriori or MAP) estimator for $\theta$ is found by minimizing $U(\bar{b}, \theta)$ (Eq. (3)) with respect to $\theta$. The complete algorithm is therefore: 
1. Compute an initial estimate $\bar{b}=b^{(0)}$ for the segmentation indicator variables and set $t=0$;

2. Until convergence do steps (3-4):

3. MAP-step: compute $\theta^{(t+1)}=\arg \min _{\theta} U(\bar{b}, \theta)$;

4. MPM-step:

a) Compute the estimators $p_{k}(r)$ for the posterior marginals $\pi_{k}(r), r \in L$, $k=1, \ldots K$ solving the linear system resulting from the minimization of (51).

b) Set $\bar{b}_{k}(r)=1$, if $p_{k}(r)>p_{k^{\prime}}(r)$ for $k^{\prime} \neq k$, and $\bar{b}_{k}(r)=0$, otherwise; set $t:=t+1$

The convergence of the algorithm follows from the facts that $Q$ is bounded below (it is always non-negative) and that it cannot increase in one full iteration; it could converge to a limit cycle, but in practice it is found to converge to a fixed point. Note that this algorithm is more robust than EM [2] with respect to errors in the estimation of the posterior marginals $\left\{\pi_{k}(r)\right\}$, because only the location of the mode of each distribution $\pi(r)$ is of consequence.

\subsection{Parametric Models of the Intensities in Each Tissue Class}

To complete the specification of the approach we are proposing, it is necessary to define the parametric models that represent the spatial variation of the intensity within each tissue class. This intensity, in an MR image, is usually not a constant, due to the variations in the magnetic properties of the biological tissues, irregularities in the magnetic fields, operating conditions of the MR equipment, etc. In this paper, instead of the usual assumption of a multiplicative bias field that modulates a class-dependent constant intensity, we use a more general approach in which the variable intensity for each class is separately modeled. Here, we use spline models with a Gibbsian prior $P(\theta)$ that imposes a controlled smoothness constraint. This is equivalent to a finite element approximation to a membrane spline. In particular, we define the model $\Phi\left(r, \theta_{k}\right)$ as:

$$
\Phi\left(r, \theta_{k}\right)=\sum_{j=1}^{m} N_{j}(r) \theta_{k j}
$$

where $\left\{N_{j}\right\}$ are the classical trilinear interpolation functions used for the 8-node Lagrangian element [25], and the parameters $\theta_{k r}$ correspond to the height of the membrane at the nodes of the finite element mesh.

The smoothness constraint is specified in the form of a Gibbs distribution:

$$
P\left(\theta_{k}\right)=\frac{1}{Z} \exp \left[-\eta \int_{L}\left|\nabla \Phi\left(r, \theta_{k}\right)\right|^{2} d r\right]
$$

where $Z$ is a normalizing constant and $\eta$ is a parameter. This scheme allows one to model arbitrary smooth shapes, while retaining sufficient control on the smoothness of each model (given by the positive parameter $\eta$ ). Substituting (9) 
and (10)into (3) and setting the partial derivatives of $U$ with respect to the parameter vector $\theta_{k}$ equal to zero, one obtains a system of linear equations whose solution gives the optimal parameters. In this way, the whole procedure involves only the solution of linear systems (in both the MAP and MPM steps) and is, therefore, computationally efficient.

\subsection{Prior Class Probabilities and Automatic Initialization}

To improve the performance of a segmentation method, we should take advantage of domain-dependent relevant information. In the particular case of brain MRI, we consider the fact that the location of the main anatomical structures is approximately the same in most subjects, if the brain is brought to a standard position and scale. Therefore, it should be possible to incorporate into the segmentation procedure, prior information about the approximate location of the different tissue classes. This information may then be used to provide an initial approximate segmentation to initialize the algorithm (step 1 in section $2.2)$ and also to estimate the prior class probabilities for each voxel $\left(q_{k}(r)\right.$ in Eq. (3)). The class location information may be obtained from statistical studies, in which hand-segmented MRI's for a given population of subjects are brought to a standard position, so that the frequencies with which each tissue class appears at each standard voxel location can be computed. One such study, based on a population of 151 normal subjects, is available in [12, and is the one we used in our experiments. To incorporate this information in our procedure, it is necessary to find a transformation that maps the standard anatomical model (to which the intensity distribution is referred) into the specimen to be segmented. Here, we use the level-set based PDE procedure described in [4] to estimate the transformation. Once this transformation is obtained, it is used to: segment the brain from non-brain tissue ("peeling" the brain); to map the prior probabilities to the specimen space and to find a good approximate segmentation to initialize the procedure.

\subsection{Complete Segmentation Procedure}

In summary, the complete segmentation procedure we are proposing consists of the following steps:

1. Find the complete transformation $T$ that maps the standardized (atlas) space into the specimen space using the 2-step procedure of section 2.4, and peel the brain using transformation $T$.

2. Obtain the initial segmentation $b^{(0)}$ from the anatomical model mapped into the specimen space using $T$.

3. Map the empirical frequency field using $T$ and compute the prior class probability field using $q_{k}(r)=\alpha f_{k}(r)+(1-\alpha) / K$, where, $K$ is the number of classes and $\alpha \in[0,1]$ weights the influence of the frequencies.

4. Apply the MAP-MPM algorithm of section 2.2 until convergence. 
In the case of multi-band images (e.g., T1, T2 and PD weighted MRI), the individual images have to be registered separately, and a different membrane model has to be adjusted for each band in the MAP step in a decoupled manner. One finds, however, a unique segmentation in each MPM step.

This procedure has a number of parameters: $\gamma$ in equation (1); $\lambda$ in Eq. (5) and $\eta$ in Eq. (10). The best value for these parameters has to be hand-picked using a trial and error procedure on a test image. Once these values are found, however, they may be used in different data sets without need of further tuning, since the performance of the method is quite insensitive to their precise value. The values we have used in all the experiments reported in the next section, both for synthetic and real data, are: $\gamma=1 ; \lambda=0.1$ and $\eta=1000$. The finite element mesh size used in all our experiments to be described in the next section is $(32 \times 32 \times 8)$.

\section{Experimental Validation}

To validate the performance of our method, we performed 2 sets of experiments, one on simulated MR and another on real MR brain scans. In the first set, we used the simulated MR images of the head generated using the BrainWeb MR simulator 1218 . Since in this case, the anatomical model (ground truth) available, it is possible to obtain a quantitative assessment of the performance of the algorithm, under different conditions (different levels of noise, spatial inhomogeneities and contrast). We considered the following cases: (i) T1 weighted images with 1 through $9 \%$ noise levels and no spatial inhomogeneity. (ii) Same as (1), with $40 \%$ spatial inhomogeneity. (iii) Multi-band (T1, T2 and PD weighted) data with 1 through $9 \%$ noise and $40 \%$ spatial inhomogeneity. Only results for the latter case are shown in this paper for the sake of brevity.

Since these experiments were also reported in [23, in order for the results to be meaningfully compared, we used the performance index reported therein. The results are shown as plots at the top of the Figure (11). For the sake of brevity, only gray matter segmentation results are shown here but results for white matter and CSF depict similar superior performance as well. As one can see, the proposed method shows an excellent and stable performance, outperforming the best results (to our knowledge) reported to date in literature. Showing good performance with simulated data, however, is not sufficient to validate a segmentation procedure. It is also very important to test it with real images and compare it with other published methods. This comparison is difficult to do, because most of the published methods work with different data sets. To overcome this problem, we use the 20 normal MR brain data made publicly available on the world wide web by the Center for Morphometric Analysis at Massachusetts General Hospital. In addition, manual expert segmentations and performance results from five automatic segmentation methods are also provided at this site [5], making it convenient to compare our results with those reported by the five automatic methods. The 20 coronal scans of this data set were chosen because they have been used in published volumetric studies in the past, and because they have 

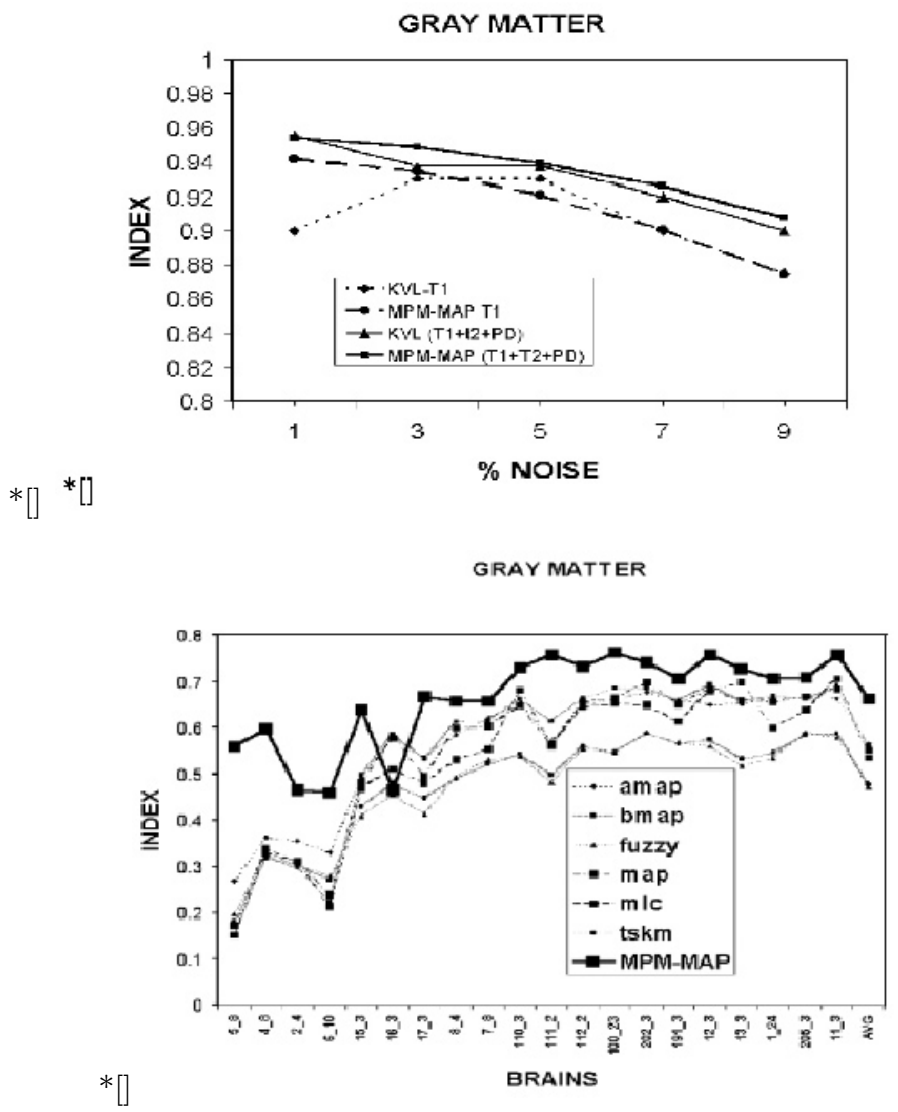

Fig. 1. Performance indices for the segmentation of: (Top row) MRI simulated data (from the brainweb) with $40 \%$ spatial inhomogeneities, using our algorithm and the one in [21] for gray matter; (Bottom row:) real MRI data from the MGH database. The results for white matter and CSF are similar.

various levels of difficulty; the worst scans have low contrast and relatively large spatial inhomogeneities. Hence, these test images permit a standardized mechanism for testing the sensitivity of a proposed segmentation method to signal to noise ratio, contrast to noise ratio, shape complexity, degree of partial volume effect, etc. The repository also contains performance indices that measure the amount of overlap between the expert hand-guided segmentation and a collection of automatic methods. The index in this case is the Tanimoto coefficient [27.

A final validation comes from the positive opinion of practicing neuro- scientists.

We applied our method to the twenty complete head MR scans in this data set, using the same parameter values in all cases, so that it may, in effect, be considered an automatic procedure. The results are summarized in table (1) 

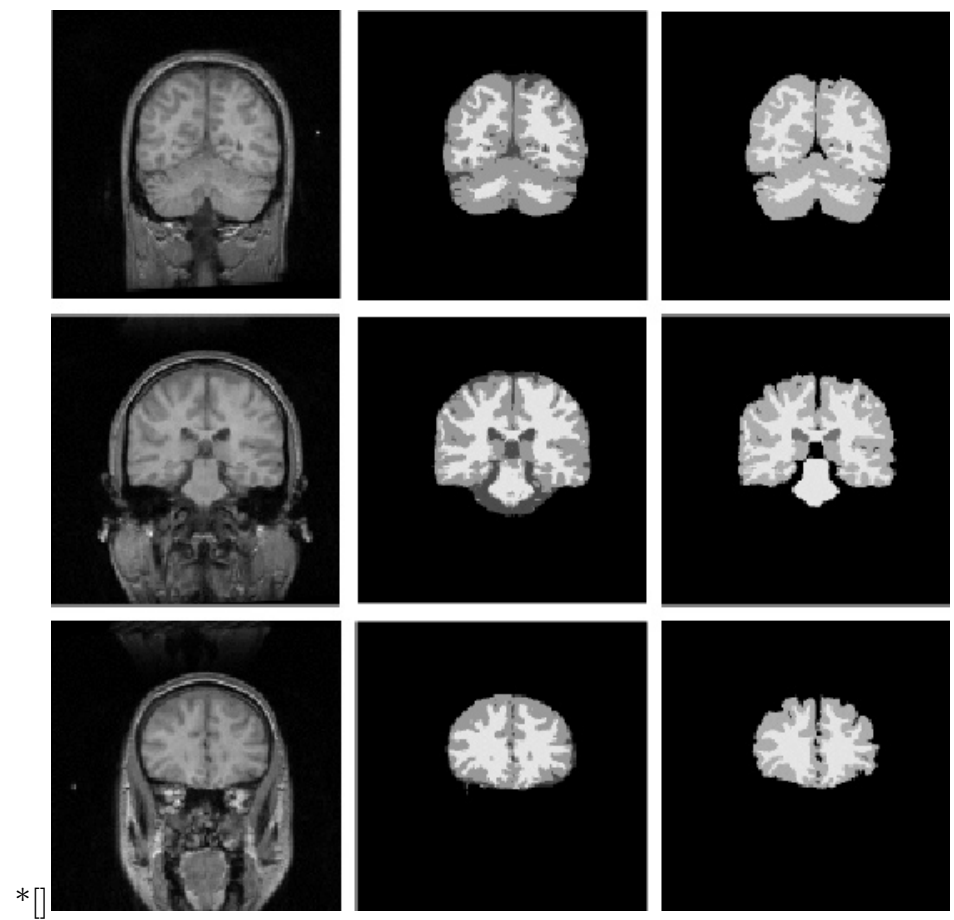

Fig. 2. Sample images from the MGH database. On each row: left: original image; center: MPM-MAP automatic segmentation; right: expert hand-guided segmentation (see text for details).

and the bottom plots in figure (1). Once again, only gray matter segmentation performance is depicted here as in the simulated data case for the sake of brevity. The dimensions of the image stacks were $256 \times 256 \times 64$ voxels, and the average total processing time (including registration for peeling the skull and non-brain material and segmentation) was 29 minutes on a single processor of an SGI ONYX machine. As one can see, these results are significantly better than all other reported methods (see [5] for details on these other methods). Figure (2) depicts a sample of our segmentation results, together with the corresponding expert (ground truth) segmentation. We used an intensity value of 255, 170 and 85 to depict the white matter, gray matter and CSF pixels respectively.

\section{Discussion and Conclusions}

The Bayesian estimation framework is very convenient for MRI segmentation, because it permits the inclusion of explicit models for the spatial coherence and location of the different tissues, as well as for the spatial inhomogeneities of the corresponding image intensities caused by the bias field in the magnetic field of the MR instrument. For Bayesian-based methods to be effective, however, it is 
Table 1. Average Overlap (performance indices) between manually-guided segmentations and various methods for the 20 brain scans of the MGH data base.

\begin{tabular}{|c|c|c|}
\hline Method & Gray Mat. & White Mat. \\
\hline \hline adaptive MAP & 0.564 & 0.567 \\
\hline biased MAP & 0.558 & 0.562 \\
\hline fuzzy c-means & 0.473 & 0.567 \\
\hline Maximum a Posteriori Probability (MAP) & 0.550 & 0.554 \\
\hline tree-structure k-means & 0.477 & 0.571 \\
\hline Maximum-Likelihood & 0.535 & 0.551 \\
\hline MPM-MAP & 0.662 & 0.683 \\
\hline Manual (4 brains averaged over 2 experts) & 0.876 & 0.832 \\
\hline
\end{tabular}

crucial to have: realistic spatially varying models for the intensity of each class; efficient and accurate ways for computing optimal segmentations given these intensity models and robust and precise registration procedures, so that prior information about the location of anatomical structures may be incorporated.

We have presented a fully automatic method that incorporates these features, and therefore depicted a superior performance over existing methods reported in literature.

Acknowledgments. J.L. Marroquin and S. Botello were supported in part by a grant from Conacyt and Calderon by Concyt and PROMEP, Mexico. B.C. Vemuri was in part supported by grants NSF IIS9811042 and NIH RO1-RR13197 respectively. The authors are grateful to Drs. T. Harmony and A. FernandezBouzas for their advice on the experimental validation of the method.

\section{References}

1. Evans A.C., Collins D.L., Mills S.R., Brown E.D., Kelly R.L., and Peters T.M. 3d statistical neuroanatomical models from 305 mri volumes. In Proc. IEEE Nuclear Sc. Symp., Med. Imaging, pages 1813-1817, 1993.

2. Dempster A.P., Laird N.M., and Rubin D.B. Maximum likehood from imcomplete data via the em algorithm. J. Roy. Stat. Soc. B, 39:1-38, 1977.

3. J. Besag. Spatial interaction and statistical analysis of lattice systems. Journal of Royal Statistical Society B, 36:192-236, 1974.

4. Vemuri B. C., Ye J., Chen Y., and Leonard C. M. A level-set based approach to image registration. In IEEE Workshop on MMBIA, pages 86-93, June 2000.

5. Cocosco C.A., Kollokian V., Kwan R.K., and Evans A.C. Brainweb: Online interface to a $3 \mathrm{~d}$ mri simulated brain database. Neuroimage, 5(4):part2/4, S425 (images available on www.bic.mni.mcgill.ca /brainweb/), 1997.

6. H. E. Cline, C. L. Doumulin, H. R. Hart, W. E. Lorensen, and S. Ludke. 3-d reconstruction of the brain from magnetic resonance images using a connectivity algorithm. Magnetic Resonance Imaging, 5:345-352, 1987.

7. M. E. Brummer et.al. Automatic detection of brain contours in mri data sets. IEEE Trans. on Med. Imaging, 12:153-166, 1993. 
8. Maes F., Collignon A., Vandermeulen G., Marchal G., and Suetens P. Multimodality image registration by maximization of mutual information. IEEE Trans. on Med. Imaging, 16:187-198, 1997.

9. Geiger G. and Girosi F. Parallel and deterministic algorithms for mrfs: Surface reconstruction and integration. IEEE TPAMI, 12:401-412, 1991.

10. Gerig G., Martin J., Kikinis R., Kubler O., Shenton M., and Jolesz F. Automatic segmentation of dual-echo $\mathrm{mr}$ head data. In XII Intl. Conf. on IPMI, pages 175187, 1991.

11. W.M. Wells III, W.E.L.Grimson, R. Kikinis, and F. A. Jolesz. Adaptive segmentation of mri data. IEEE Trans. on Med. Imaging, 15:429-442, Aug. 1996.

12. Ashburner J., Friston K., Holmes A., and Poline J.B. Statistical parametric mapping. Technical report, The Wellcome Dept. Cognitive Neurology, Univ. College, London, U.K. Available: http://www.fil.ion.ucl.ac.uk/spm/, 2000.

13. Marroquin J., Mitter S., and Poggio T. Probabilistic solution of ill-posed problems in computational vision. J. Am. Stat. Asoc., 82:76-89, 1987.

14. Marroquin J., F. Velasco, Rivera M., and M. Nakamura. Gauss-markov measure field models for low-level vision. IEEE TPAMI, 23:337-348, 2001.

15. Zhang J. Mean field theory in em procedures for mrf's. IEEE Trans. on Sig. Proc., 40:2570-2583, 1992.

16. Rajapakse J.C. and Krugge F. Segmentation of mr images with intensity inhomogeneities. Image and Vision Computing, 16:165-180, 1998.

17. M. Joliot and B. M. Majoyer. Three dimensional segmentation and interpolation of magnetic resonance brain images. IEEE Trans. on Med. Imaging, 12(2):269-277, 1993.

18. Held K., Kopsa E.R., Krause B.J., Wells W.M., Kikinis R., and Müller-Gartner H.W. Markov random field segmentation of brain $\mathrm{mr}$ images. IEEE Trans. on Med. Imaging, 16(6):878-886, 1997.

19. T. Kapur, W.E.L.Grimson, W. M. Wells III, and R. Kikinis. Segmentation of brain tissue from magnetic resonance images. Med. Image Aanalysis, 1(2):109-127, 1996.

20. Yuille A. L. and Kosowsky. Statistical physics algorithms that converge. Neural Computation, 6(3):341-356, 1994.

21. R. Leahy, T. Herbert, and R. Lee. Application of markov random fields in medical imaging. In IPMI, pages 1-14, 1989.

22. K. V. Leemput, F. Maes, D. Vandermeulen, and P. Suetens. Automated modelbased bias field correction of $\mathrm{mr}$ images of the brain. IEEE Trans. on Med. Imaging, 18(10):885-896, 1999.

23. K. V. Leemput, F. Maes, D. Vandermeulen, and P. Suetens. Automated modelbased tissue classification of $\mathrm{mr}$ images of the brain. IEEE Trans. on Med. Imaging, 18(10):897-908, 1999.

24. Atkins M.S. and Mackiewich B.T. Fully automatic segmentation of the brain in mri. IEEE Trans. on Med. Imaging, 17(1):98-107, 1998.

25. Zienkiewicz O.C. and Taylor R.L. The Finite Element Method. McGraw Hill, 1989.

26. T. N. Pappas. An adaptive clustering algorithm for image segmentation. IEEE Trans. on Signal Processing, 40(4):901-914, 1992.

27. Duda R.O., Hart P.E., and Stork D. G. Pattern Classification. Wiley Interscience, New York, 2001.

28. Geman S. and Geman D. Stochastic relaxation, gibbs distributions and the bayesian restoration of images. IEEE TPAMI, 6:721-741, 1984.

29. Kirkpatrick S., Gelatt C.D., and M.P. Vecchi. Optimization by simulated annealing. Science, 220(4598):671-680, 1983. 
30. Sandor S. and Leahy R. A 3d morphological algorithm for automated labeling of the cortex in magnetic resonance brain images. In AAAI Spring Symp. on Applications of Compu. Vision in Medical Image Processing, 1994.

31. Hofmann T., Puzicha J., and Buhmann J. M. Unsupervised texture segmentation in a deterministic annealing framework. IEEE TPAMI, 20(8):803-818, 1998.

32. U. Tiede T. Schiemann and K. H. Hohne. Segmentation of visible human for high quality volume-based visualization. Medical Image Analysis, 1(4):263-270, 1996. 